\title{
Robust Recommendation Algorithm based on Metadata Fusion
}

\author{
Gao Feng \\ College of computer, Changchun Normal University, \\ Jilin 130032, China \\ Gaofengg2014@126.com
}

\begin{abstract}
The metadata information of users and items for enhancing the recommendation system robustness has important valuable. Following this design philosophy, this paper first presents the user suspects assessment strategy based on Probabilistic Latent Semantic Analysis, the user suspected sexual and generic items such as meta-information to model parameters and Logistic Regression way into Bayesian probabilistic matrix factorization (BPMF) model, and then proposes Metadata-enhanced Variational Bayesian Matrix Factorization (MVBMF), designed a model of incremental learning strategy based on robust linear regression, in order to reduce the demand for model rebuilding. Experimental results show that MVBMF can effectively defend against shilling attacks and also has a high level of performance for strong and weak generalization.
\end{abstract}

Keywords: Recommender system; shilling attack; robust recommendation; MVBMF; matrix factorization

\section{Introduction}

In this paper, the metadata information of users and items have important role to improve the robustness of the algorithm. Committed to develop a robust model based on recommendation algorithm, to integrate metadata information through probability, enhance the model robustness of shilling attack.

In general, scoring matrix $R_{I \times J}$ is only information source of recommendation algorithm, recommendation algorithm analyzes mining score matrix, to recommend to user information. However, the shilling attack resulted in a large number of false will damage the credibility of scoring matrix. If recommendation algorithm can absorb the attacker to tamper the system metadata information, for example, the metadata information of users and items is bound to enhance the robustness of recommendation system [1]. Following this design philosophy, this paper proposes Metadata-enhanced Variational Bayesian Matrix Factorization (MVBMF) model. The main ideas include:

A. To analyze user rating behavior using the Probabilistic latent semantic analysis (PLSA) method, proposed suspicion of user evaluation strategies based on PLSA.

B. the user suspected sexual and generic items such as meta-information to model parameters and Logistic Regression way into Bayesian probabilistic matrix factorization (BPMF) model. C. Based on robust linear regression technique to design incremental model, to reduce the model reconstruction needs.

Experiments show MVBMF model effectively overcome the existing limitations of the robust collaborative recommendation technology, with a strong attack tolerance, in case of weak generalization, to ensure the robustness of recommendation system. 


\section{Metadata-enhanced Variational Bayesian Matrix Factorization model (MVBMF)}

Although the meta-information has the potential to improve the robustness of the value of recommender systems, but not all of the metadata information can be directly used without screening. In Recommendation system, metadata formation items (name, type, origin, etc.) is responsible for maintaining system. Authenticity is higher, can be used directly. Metainformation of Users (age, sex, occupation, etc.) is filled when user registers, value is limited. Because regardless of whether the attacker has provided false information privacy reasons and motives. However, the user's rating behavior implies the user meta-information of objective existence. User suspect information to ensure the robustness of the important factors of MVBMF model. To reveal the special meta-information, this paper proposes user Suspicion evaluation strategy based on PLSA.

\subsection{Users Suspicion Assessment}

PLSA is used in [2] shilling attack detection. In this paper, it will be used to assess the suspicion of the user, not hard to determine the two type of user.

PLSA is a Bayesian network model can reveal the co-occurrence data between latent semantic relations. In recommendation system, co-occurrence data (user, item) is considered to associate with the implicit generic variables $s$.PLSA assumes that $s$ is given, user and item conditional independence, $p($ user, item $\mid s)=p($ user $\mid s) p($ item $\mid s)$. This means that $s$ is (user,item) produced antecedents, different classes is corresponding to different sampling production rules, and (user,item) quality directly depends on the category $s$.

Parameters $p(s), p($ user $\mid s), p($ item $\mid s)$ of PLSA can be used the following Expectation maximization (EM) steps of the solution, consist of two main steps[3]:

Expectation step (E-step):

$$
p(s \mid \text { user, item }) \propto p(s) p(\text { user } \mid s) p(\text { item } \mid s)
$$

Maximization step (M-step):

$$
\begin{aligned}
& p(\text { item } \mid s) \propto \sum_{\text {user } \in U} R(\text { user }, \text { item }) p(s \mid \text { user }, \text { item }) \\
& p(\text { item } \mid s) \propto \sum_{\text {user } \in I} R(\text { user }, \text { item }) p(s \mid \text { user }, \text { item })
\end{aligned}
$$

After the two step iterative parameter initialization and convergence, local optimal solutions of parameters can be obtained.

If there is shilling attack, attack classes will be explained by the class $s^{\text {att }}$. At the same time, the attacker selected randomly filling items, leading to the points of interest of extreme dispersion. Therefore, this paper believes that uncertainty entropy of attack class in selecting scoring item must transcend all real user class. Formally, let the random variables $Q^{s} \square p($ item $\mid s)$, you can locate the attack class:

$$
s^{\text {att }}=\arg \max \mathrm{H}\left[\mathrm{Q}^{s}\right]
$$

Which, the entropy function $\mathrm{H}[\mathrm{x}]=-\sum_{x} p(x) \ln p(x) . \theta_{i}$ of user $_{i}$ Suspicion is the probability of user $_{i}$ membership attack class:

$$
\theta_{i}=p\left(s^{\text {att }} \mid \text { user }_{i}\right) \propto p\left(\text { user }_{i} \mid s^{\text {att }}\right) p\left(s^{\text {att }}\right)
$$


Experiments show that $\theta$ can be a good indication user's suspicion. For example, mean attack of $p^{\text {att }}=10 \% p^{\text {fill }}=9 \%$ injects into MovieLens100K dataset. As shown in Figure1, $\theta$ value of attacker was significantly greater than real users, and the latter mainly concentrated in the vicinity of 0 , and the discrimination is obvious.

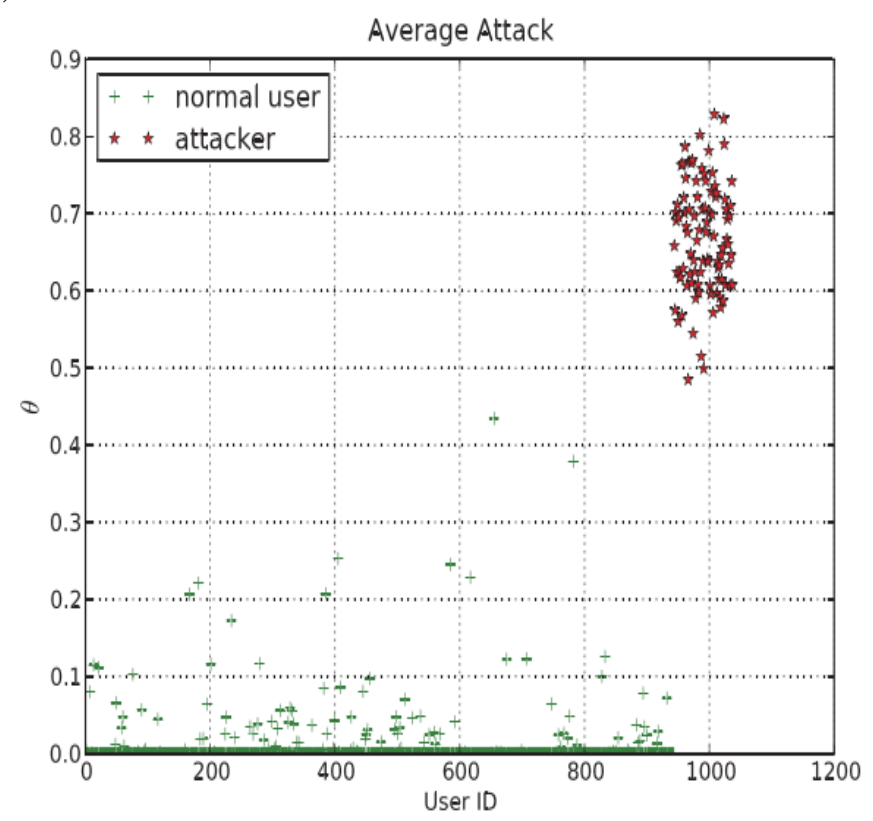

Figure 1. User Suspicion

In order to avoid the local extreme of EM algorithm to converge poor, this paper adopts a heuristic strategy.

\subsection{The Formal Description of MVBMF}

In essence, MVBMF is a matrix decomposition model. Matrix decomposition is the efficiently solution to collaborative recommendation problems. Typically such as EMSVD [4] and BPMF [5]. MVBMF inherits the BPMF infrastructure, and in accordance with integrate metadata information necessary to the corresponding expansion.

BPMF belongs to the Probabilistic generative model. Bayesian inference perspective to view matrix factorization problem, make the probability distribution assumptions for model variables [6]. Figure 2 is the probability graph model of BPMF. The random variable $R_{i j}, U_{i}, V_{j}, \mu_{u}, \wedge_{u}, \mu_{v}, \wedge_{v}$ respectively with the following distribution:

$$
\begin{aligned}
& p\left(R_{i j} \mid U_{i}, V_{j}\right)=N\left(R_{i j} \mid U_{i}^{T} V_{j}, \sigma^{2}\right)^{I_{i j}} \\
& p\left(U_{i} \mid \mu_{u}, \wedge_{u}\right)=N\left(U_{i} \mid \mu_{u}, \wedge_{u}^{-1}\right) \\
& p\left(V_{j} \mid \mu_{v}, \wedge_{v}\right)=N\left(V_{j} \mid \mu_{v}, \wedge_{v}^{-1}\right) \\
& p\left(Y_{t} \mid k\right)=N\left(\mu_{t} \mid \mu_{0}\left(\beta \wedge_{t}\right)^{-1} w\left(\wedge_{t} \mid v_{0}, \wedge_{0}\right)\right.
\end{aligned}
$$

Which $k=\left\{\mu_{0}, v_{0}, \beta, \wedge_{0}\right\}, Y_{t}=\left\{\mu_{1}, \wedge_{t}\right\}, t \in\{u, v), I_{i j}$ represents $R_{i j}$ missing, missing is 0 , otherwise is $1 . U_{i}$ and $V_{j}$ is called feature vector. Represent the preferences features of user $_{i}$ and the generic features of item $_{j}$. 


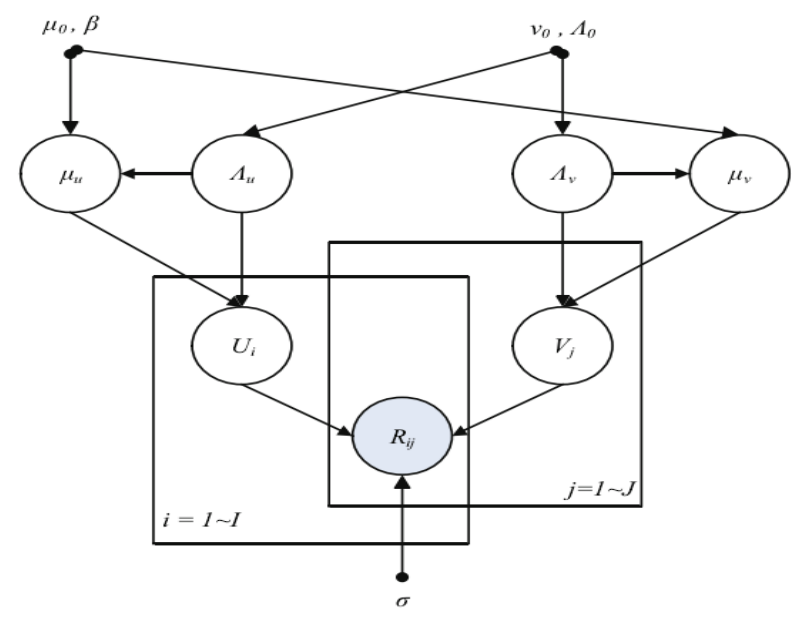

Figure 2. Graphical Model of Bayesian Probabilistic Matrix Factorization

Figure 3 is the probability graph model of MVBMF, in accordance with the following distribution of each random variable:

$$
\begin{gathered}
P\left(G_{t} \mid v_{t}, \wedge_{t}\right)=\omega\left(G_{t} \mid v_{t}, \wedge_{t}\right) \\
p\left(G_{r} \mid \alpha, \beta\right)=\operatorname{Gam}\left(G_{r} \mid \alpha, \beta\right) \\
p\left(C_{i j} \mid \lambda\right)=\operatorname{Gam}\left(C_{i j} \mid \lambda / 2, \lambda / 2\right) \\
p\left(Z_{i j} \mid \theta_{i}, x_{i j}\right)=\operatorname{Bern}\left(Z_{i j} \mid \theta_{i}, x_{i j}\right) \\
p\left(U_{i} \mid G_{u}\right)=N\left(U_{i} \mid 0, G_{u}^{-1}\right) \\
p\left(V_{j} \mid G_{v}\right)=N\left(V_{j} \mid 0, G_{v}^{-1}\right) \\
p\left(W_{k} \mid G_{w}\right)=N\left(W_{j} \mid 0, G_{w}^{-1}\right) \\
p\left(X_{j k} \mid V_{j} W_{k}\right)=\operatorname{Bern}\left(X_{j k} \mid \sigma\left(W_{k}^{T} V_{j}^{\prime}\right)\right) \\
p\left(R_{i j} \mid U_{i}, V_{j}, Z_{i j}, C_{i j}, G_{r}\right)=N\left(R_{i j} \mid U_{i}^{T} V_{j},\left(G_{r} \cdot C_{i j}\right)^{-1}\right)^{I_{i j}\left(1-Z_{i j}\right)}
\end{gathered}
$$

Which $t \in\{u, v, w\}$, Parameter $x_{i j}$ represents extreme value, extreme value is 0 , otherwise is 1

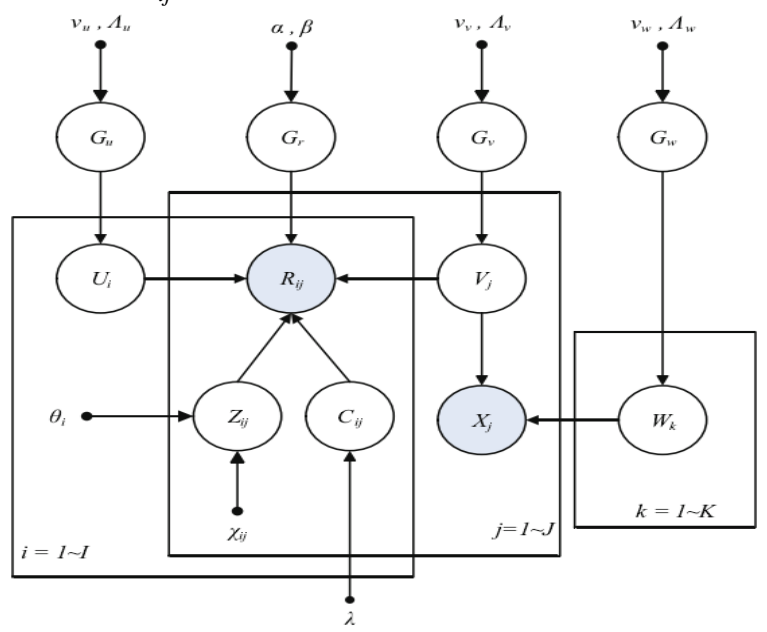




\section{Figure 3. Metadata-enhanced Variational Bayesian Matrix Factorization Model}

\subsection{The Sampling Generated Semantic of MVBMF}

The sampling process of MVBMF can be divided into four levels: Top floor:

$$
\begin{gathered}
\text { Sampling parameters } G_{t} \square W\left(G_{t} \mid v_{t}, \wedge_{t}\right) t \in\{u, v, w\} \\
\text { Sampling parameters } G_{r} \square \operatorname{Gam}\left(G_{r} \mid \alpha, \beta\right) \\
\forall k, k=1 \square K
\end{gathered}
$$$$
\text { Sample regression coefficient } W_{k} \square N\left(W_{k} \mid 0, G_{w}^{-1}\right)
$$

User layer:

$$
\forall \text { user }_{i}, i=1 \square K
$$

Sampling the user feature vector $U_{i} \square N\left(U_{i} \mid 0, G_{u}^{-1}\right)$

Item level :

$$
\forall \text { item }_{j}, j=1 \square J
$$

The sample feature vector $V_{j} \square N\left(V_{j} \mid 0, G_{v}^{-1}\right)$

$$
\forall k, k=1 \square K
$$

Sampling category $X_{j k} \square \operatorname{Bern}\left(X_{j k} \mid \sigma\left(W_{k}^{T} V_{j}^{\prime}\right)\right)$

The score level:

$$
\begin{gathered}
\forall \text { user }_{i}, i=1 \square I \\
\forall \text { item }_{j}, j=1 \square J \\
\text { Sampling parameters } C_{i j} \square \operatorname{Gam}\left(C_{i j} \mid \lambda / 2, \lambda / 2\right) \\
\text { Sampling mask indicates } Z_{i j} \square \operatorname{Bern}\left(Z_{i j} \mid \theta_{i} \cdot x_{i j}\right) \\
\text { Sampling score } R_{i j} \square N\left(R_{i j} \mid U_{i}^{T} V_{j},\left(G_{r} \cdot C_{i j}\right)^{-1}\right)^{I_{i j}\left(1-Z_{i j}\right)}
\end{gathered}
$$

\subsection{Robust Security Mechanism of MVBMF}

MVBMF provides three robust security mechanism.

First, if the score $R_{i j}$ is extreme value and the score user strong suspicion, Formula1 will make $Z_{i j}=1$ appears higher probability, when is $Z_{i j}=1, R_{i j}$ is shielded by formula3. Thus, according to the probability of extreme scores of shielding the most aggressive. Not only eliminate its negative influence on the model construction, and but also reduces the possibility of harming the real user. Obviously, user Suspicion information plays a key role in this score shielding mechanism.

Secondly, considering the variability and stability of user preferences, MVBMF believes class property has implicitly determining the true value. It should give priority to ensure the credibility of the posterior variable $\mathrm{V}$.

Finally, MVBMF also has the ability to filter noise. For the 3 formula, if $\exists i, j$ then $I_{i j}=1, Z_{i j}=0$, else $C_{i j}$ can be obtained: 


$$
\begin{aligned}
p\left(R_{i j} \mid U_{i}, V_{j}, G_{r}\right) & =\int_{0}^{\infty} p\left(R_{i j} \mid U_{i}, V_{j}, C_{i j}, G_{r}\right) p\left(C_{i j}\right) d C_{i j} \\
& =\int_{0}^{\infty} N\left(R_{i j} \mid U_{i}^{T} V_{j},\left(G_{r} \cdot C_{i j}\right)^{-1}\right) \cdot \operatorname{Gam}\left(C_{i j} \mid \lambda / 2, \lambda / 2\right) d C_{i j} \\
& =\operatorname{St}\left(R_{i j} \mid U_{i}^{T} V_{j}, G_{r}, \lambda\right)
\end{aligned}
$$

Which, $S t(\cdot)$ is t distribution. T distribution in probability model is often used in interference noise point. This is attributed to the characteristics of the Heavy tail [7-8].

\subsection{The Incremental Learning of MVBMF}

Recommendation algorithm model should also have the Weak generalization and Strong generalization capacity [9]. The former requires algorithm can predict user score of training set, which further requires the algorithm can predict user score of independent test set.

MVBMF currently has only weak generalization ability, cannot provide the service recommended for new users. Solutions is the trigger model reconstruction when a new user joins every time. But this will cause the increase of computational cost. In order to solve this problem. This paper put forward the strategy of MVBMF model incremental learning based on robust linear regression [10].

Because of the stability of item, for $\forall$ item $_{j}$, in addition to the refactoring operation of model, adding any new user will not change the feature vector $E\left[V_{j}\right]$, and the metainformation of items enhance the reliability of $E\left[V_{j}\right]$.Therefore, as long as feature vector $\hat{U}^{\prime}$ of new users user is obtained, could predict the score:

$$
R\left(\text { user' }^{\prime} \text {, item }_{j}\right)=\widehat{U}^{T} E\left[V_{j}\right]
$$

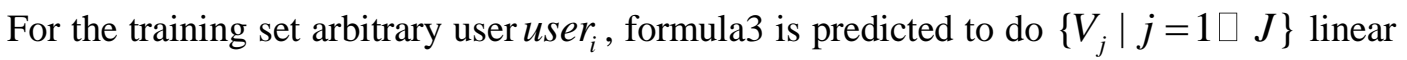
regression of all its rating. The regression coefficient $U_{i}$ is as the feature vector of user $_{i}$. The random error is in accord with t distribution. Similarly, for new user user', for the sake of its feature vector $\hat{U}^{\prime}$ and also filter out noise naturally, for user' existing ratings to predict $\left\{E\left[V_{j}\right] \mid j=1 \square J\right\}$ robust linear regression.

Let $R\left(U^{\prime}\right)$ represents the user user' index set of evaluated, can be obtained:

$$
\begin{aligned}
& r(j, \eta)=R\left(\text { user }^{\prime}, \text { item }_{j}\right)-E\left[V_{j}\right]^{T} \eta \\
& \eta^{0}=\arg \min \sum_{j \in R\left(U^{\prime}\right)}|r(j, \eta)| \\
& r e s=\left\{\mid r\left(j, \eta^{0}\right) \| j \in R\left(U^{\prime}\right) \wedge r\left(j, \eta^{0}\right) \neq 0\right\} \\
& \bar{\sigma}=\frac{\operatorname{Median}(\text { res })}{0.675} \\
& \psi(\eta)=\sum_{j \in R\left(U^{\prime}\right)} p\left(\frac{r(j, \eta)}{\bar{\sigma}}\right)
\end{aligned}
$$

The feature vector user' is the regression coefficients of M- estimate $\hat{\eta}$ :

$$
\widehat{U}^{\prime}=\widehat{\eta}=\arg \min \psi(\eta)
$$


Which, $\eta^{0}$ is least absolute deviation estimation of the regression coefficient. Algorithm sees [11]. Expansion estimator $\bar{\sigma}$ gives $\hat{\eta}$ scaling invariance. Function $p(\cdot)$ is the deciding factor of regression robustness. Using a common re-descending estimators (Two weighted estimator)

$$
\rho(x)= \begin{cases}1-\left(1-\left(\frac{x}{k}\right)^{2}\right)^{3} & |\mathrm{x}| \leq \mathrm{k} \\ 1 & |\mathrm{x}|>\mathrm{k}\end{cases}
$$

In the formula7, the necessary conditions of $\hat{\eta}$ existence:

$$
\Delta \psi(\widehat{\eta})=0=\sum_{j \in R\left(U^{\prime}\right)} p\left(\frac{r(j, \widehat{\eta})}{\bar{\sigma}}\right) E\left[V_{j}\right] r(j, \widehat{\eta})=0
$$

Using iteratively reweighted least squares algorithm [12] to calculate $\hat{\eta}$. Which, weights is $w(x)=\left(1-\left(\frac{x}{k}\right)^{2}\right)^{2} \mathrm{I}(|x| \leq k)$.As shown in Figure 4.

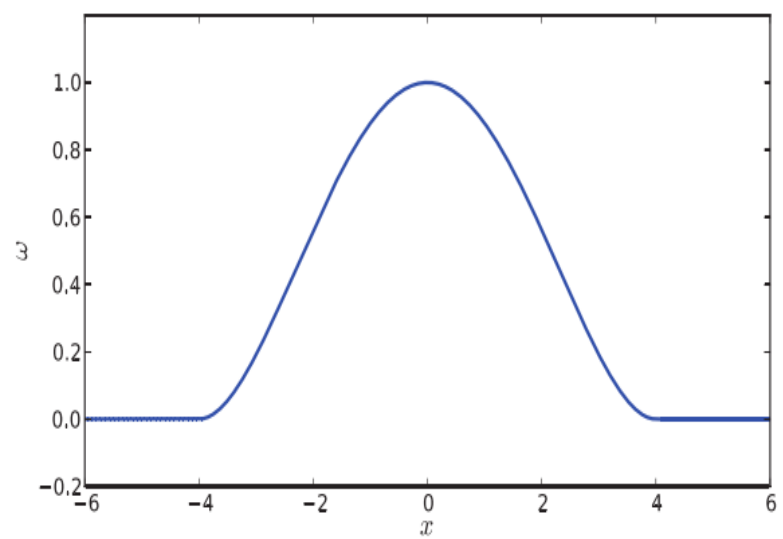

Figure 4. Weights Function of Two Weighted Estimator

Because of the $R\left(U^{\prime}\right)$ is low base, so robust linear regression process takes very little time, the computational cost is much smaller than the model reconstruction.

\section{Experimental Analysis and Results}

\subsection{Data Sets and Experimental Setup}

The experimental data sets is MovieLens100K and MovieLens1M. Two data sets of each film is one of at least 19 kinds of video types. For the MovieLens100K data set, the experiment adopts all users and items. For the MovieLens1M data set, the experiment adopts all items. But randomly choose one fifth (around 1200) users. Set the values of model parameters $v_{t}=1, \wedge_{t}=I, \alpha=8, \beta=4, \lambda=8$.

In order to validate the robustness of MVBMF model, assume that the data from original users is real users. In different the attack size of $\rho^{\text {att }}$ and filler size $\rho^{\text {fill }}$. The data set is injected into four kinds of attacks. Random attack, average attack, bandwagon attack and segment attack. Which filler size of bandwagon attack selected for the highest individual items of Popularity. Filler size of segment attack selected for the same items of five types. 
Robust quantitative measure used two indicators: Mean absolute error (MAE) and prediction error (PS).

\subsection{Dimension Selection}

MVBMF is a Bayesian model. Bayesian model complexity should not be too high or too low, otherwise, it will cause excessive and reduce the observed value. In the MVBMF model, positive integer $\mathrm{D}$ is the dimension of the user and the feature vector, directly controls the complexity of model. In order to select the appropriate D value. This paper is comparing the MAE value of MVBMF model of MovieLens100K and MovieLens1M when D is $2,5,10,15,20$.Generally, the value of $\mathrm{D}$ is small to reveal diversity the types of users and items, the value of $\mathrm{D}$ is big to be the risk of over-fitting, two categories will reduce model generalization. Generally, an optimum value of D is more moderate values associated with the modeling data. In the experiment, because the MovieLens1M data is larger than MovieLens100K. Type diversity of the user and item is more abundant, so dimension of the required should be greater than MovieLens100K. In Figure5, the experimental results are in agreement with the above analysis. In the two data sets, MVBMF at $D=5$ and $D=15$ is obtained the best accuracy. Accordingly, the following experiment, $D=5$ and $D=15$ are respectively corresponding to the data set of MVBMF and model dimension of other reference

\subsection{Weak Generalization Situation}

First, comprehensive evaluation of weak generalization ability of MVBMF in MovieLens100K data sets, and with EMSVD, RMF, VarSelect SVD, BPMF and SMVBMF is performance comparison, which, SMVBMF is a simplification of the MVBMF, delete the meta-information of user and item, leaving the rest to verify the impact of the robustness of the meta-information. The experiment adopted $4 \times 3 \times 2$ design patterns

Attack model (Random attack, Average attack, Bandwagon attack and Segment attack), different combination of the attack size of $\rho^{\text {att }}(3 \%, 5 \%, 10 \%)$ and filler size $\rho^{\text {fill }}(3 \%, 6 \%)$ corresponds to a group of experimental configuration. The final data were obtained from mean value ten independent experiments. 

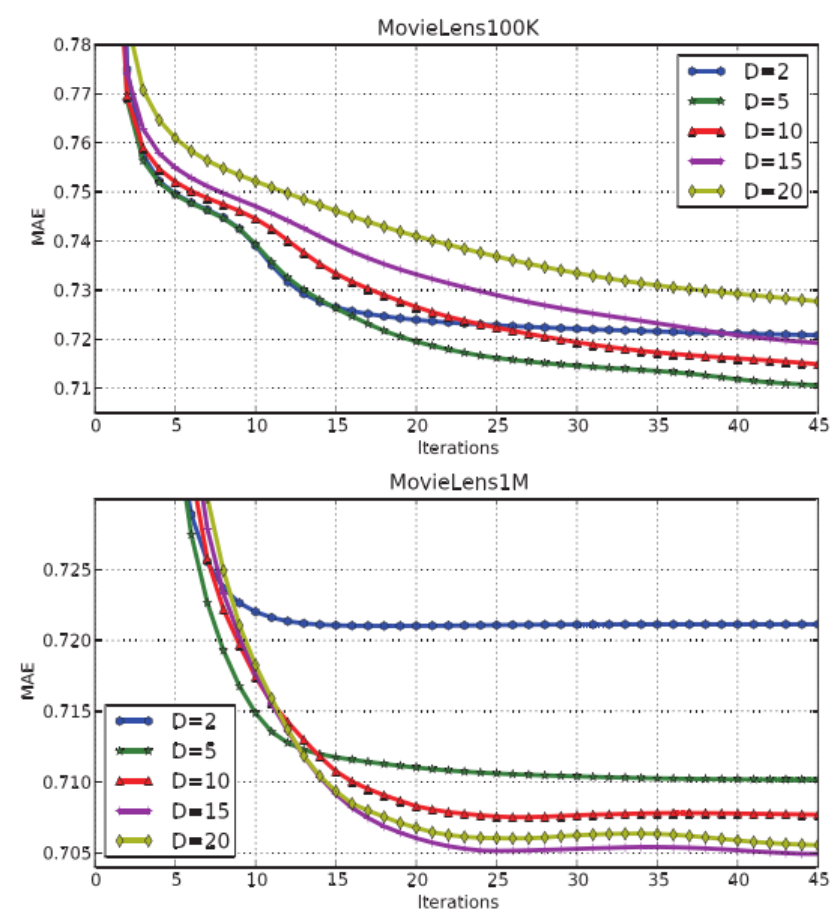

Figure 5. Dimension Selection of MVBMF

Figure 6 can be seen, in two aspects of accuracy and robustness, MVBMF is better than other models. Although in the face of random attack, average attack, bandwagon attack, VarSelect SVD robustness is weaker than MVBMF, but the former but the robustness of the former in Segment attack produces severe degradation. In particular, the robustness of the most models in Figure 6 will be significantly weakened in the face of some attacks. In contrast, the robustness of MVBMF in all four supporting attacks were maintained a higher level, has a significant advantage.
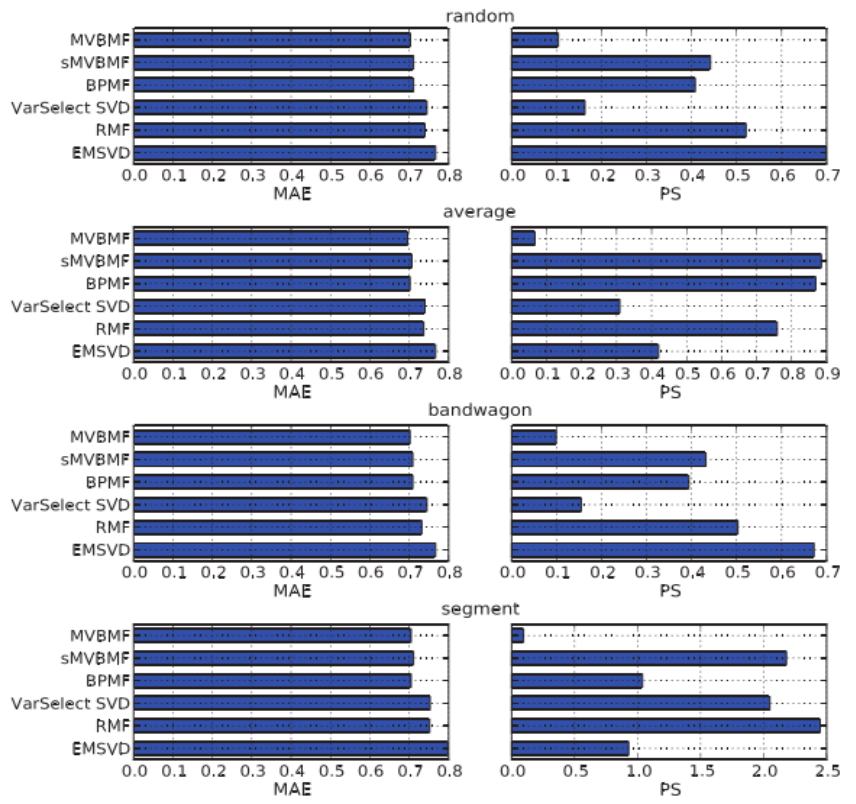

Figure 6. Performance Comparison of Different Recommendation Model 


\subsection{Strong Generalization Situation}

In order to assess MVBMF the ability to strong generalize, the MovieLens100K data set is divided into training set and test set of independent, in order to simulate the new user. The experiment adopted $3 \times 5$ design patterns. The different combination of the proportion of the training set $(20 \%, 50 \%, 80 \%)$, Attack situation (No attack, Random attack, Average attack, Bandwagon attack and Segment attack) corresponds to a group of experimental configuration. Attack parameters are $\rho^{\text {att }}=10 \% \rho^{\text {fill }}=6 \%$, experiment random retained for ten score test of each new user to evaluate model incremental learning strategy is missing a lot of score predictive ability. The final data were obtained from mean value ten independent experiments.

In theory, because the information was not involved in the new user model building, strong generalization ability of MVBMF is not weak generalization level. From table1 shows, in the $20 \%$ training set, the accuracy of MVBMF is generally poor, and only in the face of segment attack showed good robustness, the robustness in the attack scenario is not ideal, so this is necessary to reconstruct model. But in the rest of the training set ratio, MAE and PS MVBMF values were close to weak generalization situation, and with the training set increases to show decreasing trend

\section{Conclusion}

In this paper, based on introducing the evaluation strategy of user suspicion, the user suspicion and item category meta-information and Bayesian probabilistic matrix factorization model is integrating. Proposed Metadata-enhanced Variational Bayesian Matrix Factorization (MVBMF) model for robust collaborative recommendation, and designs the learning strategies of the model incremental. MVBMF model uses PLSA, Logistic regression and the variational EM method, the user suspicion and item category information brings into the matrix decomposition process, weakening malicious score for negative influence of the model variables. MVBMF has significant tolerance to the shilling attack, the strength of its ability to achieve a higher level of generalization, it can provide robust collaborative recommendation service to users.

Table 1. The Performance of Incremental Learning

\begin{tabular}{|c|c|c|c|c|c|c|}
\hline \multirow[t]{2}{*}{ Ratio the training set } & \multicolumn{2}{|l|}{$20 \%$} & \multicolumn{2}{|l|}{$50 \%$} & \multicolumn{2}{|l|}{$80 \%$} \\
\hline & MAE & PS & MAE & PS & MAE & PS \\
\hline No attack & 0.90 & $\begin{array}{ll}----- \\
\end{array}$ & 0.81 & $\begin{array}{ll}---- \\
\end{array}$ & 0.79 & $\begin{array}{l}----- \\
\end{array}$ \\
\hline Random attack & 0.88 & 1.53 & 0.80 & 0.25 & 0.79 & 0.23 \\
\hline Average attack & 0.91 & 1.47 & 0.80 & 0.27 & 0.78 & 0.24 \\
\hline Bandwagon attack & 0.87 & 1.58 & 0.81 & 0.26 & 0.78 & 0.23 \\
\hline Segment attack & 0.85 & 0.19 & 0.79 & 0.19 & 0.79 & 0.18 \\
\hline
\end{tabular}

\section{References}

[1] B. Mobasher, R. D. Burke and R. Bhaumik, et al. "Attacks and Remedies in Collaborative Recommendation”, IEEE Intelligent Systems, vol. 22, no. 3, (2007), pp. 56-63.

[2] B. Mehta and W. Nejdl, "Unsupervised Strategies for Shilling Detection and Robust Collaborative Filtering", User Modeling and User-Adapted Interaction, vol. 19, nos. 1-2, (2009), pp. 65-97. 
[3] B. Mobasher, R. Burke and J. Sandvig, "Model-based Collaborative Filtering as a Defense against Profile Injection Attacks", In Proceedings of the 21st national conference on Artificial intelligence, Boston, Massachusetts, (2006), pp. 1388-1393.

[4] S. Zhang, W. Wang and J. Ford, et al. "Using Singular Value Decomposition Approximation for Collaborative Filtering", In Proceedings of the 7th IEEE international conference on E-commerce technology, (2005), pp. 257-264.

[5] R. Salakhutdinov and A. Mnih, "Bayesian Probabilistic Matrix Factorization using Markov Chain Monte Carlo", In Proceedings of the 25th international conference on Machine learning, Helsinki, Finland, (2008), pp. 880-887.

[6] S. Williamson and Z. Ghahramani, "Probabilistic Models for Data Combination in Recommender Systems", In Proceedings of the NIPS workshop: Learning from Multiple Sources, Vancouver, Canada, (2008), pp. 1-4.

[7] M. E. Tipping and N. D. Lawrence, "Variational Inference for Student-t Models: Robust Bayesian Interpolation and Generalized Component Analysis", Neurocomputing, vol. 69, nos. 1-3, (2005), pp. 123-141.

[8] M. Svensén and C. M. Bishop, "Robust Bayesian Mixture Modelling", Neurocomputing, vol. 64, (2005), pp. $235-252$.

[9] B. Marlin, "Collaborative Filtering: A Machine Learning Perspective", Canada: University of Toronto, (2004).

[10] P. J. Huber and E. M. Ronchetti, "Robust Statistics (2nd edition)", New Jersey: John Wiley and Sons, (2009).

[11] I. Barrodale and F. D. K. Roberts, "An Improved Algorithm for Discrete Linear Approximation", SIAM Journal on Numerical Analysis, vol. 10, no. 5, (1973), pp. 839-848.

[12] J. O. Street, R. J. Carroll and D. Ruppert, "A Note on Computing Robust Regression Estimates via Iteratively Reweighted Least Squares", The American Statistician, vol. 42, no. 2, (1988), pp. 152-154.

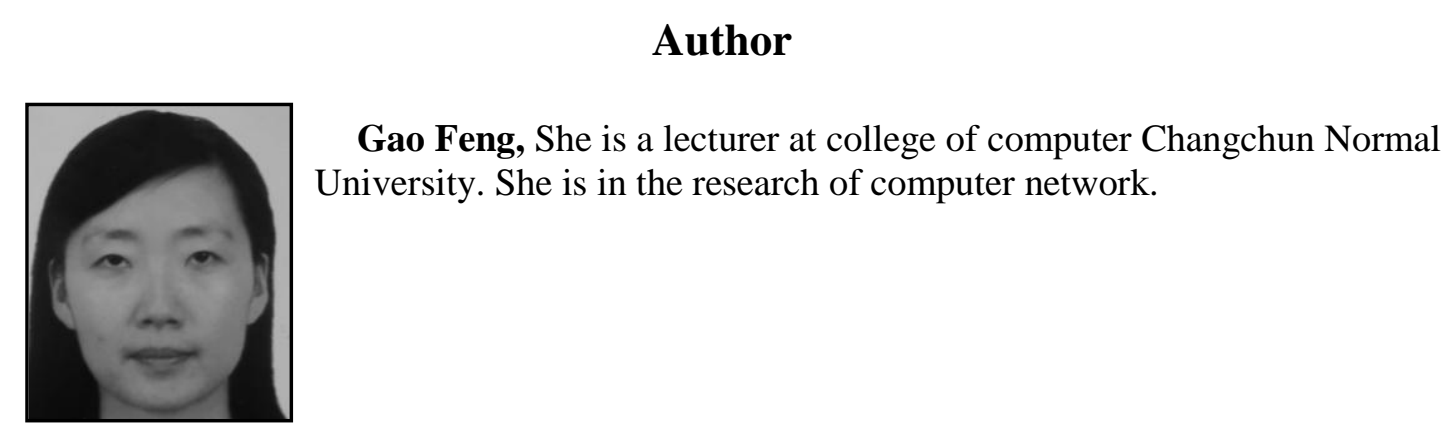


International Journal of $u-$ and e-Service, Science and Technology Vol. 7, No. 6 (2014) 